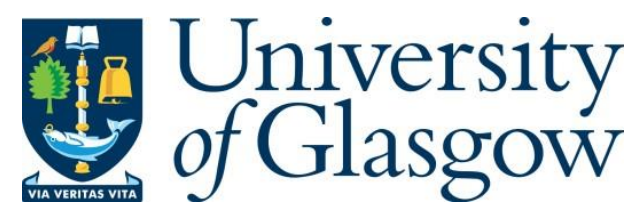

Ekti, A. R., Shakir, M. Z., Serpedin, E., Qaraqe, K. A., and Imran, M. A. (2016) On the traffic offloading in Wi-Fi supported heterogeneous wireless networks. Journal of Signal Processing Systems, 83(2), pp. 225-240.

There may be differences between this version and the published version. You are advised to consult the publisher's version if you wish to cite from it.

http://eprints.gla.ac.uk/132748/

Deposited on: 24 February 2017

Enlighten - Research publications by members of the University of Glasgow http://eprints.gla.ac.uk 


\title{
On the Traffic Offloading in Wi-Fi Supported Heterogeneous Wireless Networks
}

\author{
Ali Riza Ekti • Muhammad Zeeshan \\ Shakir • Erchin Serpedin • Khalid A. \\ Qaraqe · Muhammad A. Imran
}

Received: date / Accepted: date

\begin{abstract}
Heterogeneous small cell networks (HetSNets) are becoming an integral part of the next generation wireless networks (NGWNs), where small cell base stations (SBSs) are expected to utilize wireless-fidelity (Wi-Fi) access points (APs) and device-to-device (D2D) enabled links. HetSNets comprise several low power, low cost SBSs, D2D enabled links and Wi-Fi APs to support the existing macrocell infrastructure, decrease over the air signaling and energy consumption, and increase network capacity, data rate and coverage. This paper presents an active user dependent path loss (PL) based traffic offloading (TO) strategy for HetSNets and a comparative study on two techniques to offload the traffic from macrocell to small cell: PL and signal-to-interference ratio (SIR) based strategies. To quantify the improvements, the PL based strategy against the SIR based strategy is compared while considering various macrocell and small cell coverage areas and traffic-types. On the other hand, offloading in a dense urban setting may result in overcrowding the small cells. Therefore, hybrid traffic-type driven hybrid offloading technologies such as $\mathrm{Wi}-\mathrm{Fi}$ and $\mathrm{D} 2 \mathrm{D}$ were proposed to en route the delay tolerant applications through Wi-Fi APs and D2D links. It is necessary to illustrate the impact of daily user traffic profile, small cell access schemes and traffic-type while deciding how much of the traffic should be offloaded to small cells. It is also important to assess the number of active small cells based on the active user population factor (AUPF), since AUPF defines the ratio of the number of active
\end{abstract}

This work was supported by NSF Grant: CCF-1318338.

A.R. Ekti, E. Serpedin

Department of Electrical and Computer Engineering, Texas A\&M University, College Station, TX, 77843-3128, U.S.A.

E-mail: arekti@tamu.edu, serpedin@ece.tamu.edu

M.Z. Shakir, K.A. Qaraqe

Department of Electrical and Computer Engineering, Texas A\&M University at Qatar, Education City, 23874, Doha, Qatar

E-mail: muhammad.shakir@qatar.tamu.edu,khalid.qaraqe@qatar.tamu.edu

M.A. Imran

Center for Communication Systems Research (CCSR), University of Surrey, Guildford, UK

E-mail: m.imran@surrey.ac.uk 
mobile users and the number of total mobile users, and it also describes the daily user traffic profile. In this context, we propose that the population of smallcells is strictly depending on the traffic load due to active mobile users, which is a random variable and time--varying. Therefore, in this paper, the AUPF, small cell access scheme and traffic-type are included into a PL based TO strategy to minimize the congested macrocell traffic.

Keywords Heterogeneous networks, Small cells, Wi-Fi, WiGig, Wi-Fi Direct, Traffic offloading, Active user population factor, and Path loss

\section{Introduction}

In terms of wireless communications system evolution, there are two major distinct trends: (a) improve the existing capabilities, and (b) extend and insert new features into the existing structures. When the first item is considered, it is clear that the improvement of already existing capabilities is a must for the network operators. The agile evolution of wireless communications systems has led to the emergence of new concepts in terms of quality of service (QoS) and system efficiency for the next generation wireless networks (NGWNs) such as $5 \mathrm{G}$ networks [1]. NGWNs will operate at higher frequencies and in the unlicensed spectrum due to the support for more bandwidth, high data rates and reduced interference. However, higher frequency transmissions suffer from greater propagation losses. In addition, there is a continuous demand for new applications and services. An example in this regard is that of wireless cellular communications systems which were initially designed to address voice based communications. In the meantime, wireless communications systems have evolved into information sharing networks where people are connected uninterruptedly via social networking, video broadcasting, peer-to-peer (P2P) sharing and online gaming.

Currently, the wireless industry witnesses explosive growth. The number of mobile subscribers, which was 4.5 billion in 2012 , is expected to reach 7.6 billion by 2020 , and the mobile data traffic volume, which was 45 million terabyte (TB)/year in 2012, is expected to reach 623 million TB/year by 2020 [2]. Furthermore, the data traffic patterns depend upon the time of the day and the density of users in a particular location. For instance, the data traffic is variable during a 24 hour period as it is smaller during night time when the users are inactive. On the other hand, the data traffic will increase exponentially during the day time, since the number of active users and data transmission rates escalate. Another effectual aspect of data traffic density is the geographical location of the users. Traffic load is predicted to rise rapidly in highly populated areas such as downtowns, shopping centers and other public places. Therefore, it is necessary to take into account the active user population factor (AUPF) along with other factors. Rapidly accumulating device diversity and data traffic aggregated with the above mentioned factors make load management and network planning problems more complicated.

Network operators need to maintain the QoS while experiencing insatiable traffic growth and demands. Therefore to accomplish this, the researchers are seeking new architectural deployment strategies that can resolve the inevitable customer demands for high data traffic and new services. To cope up with these issues, numerous approaches are under consideration: (i) utilizing the radio frequency (RF) 
spectrum more efficiently, e.g., traffic balancing between unlicensed and licensed spectrum; (ii) modifying the existing mobile backhaul systems to transfer the data expeditiously; (iii) altering cell size in accordance with the traffic load conditions and the received interference from neighboring cells. One of the main design criteria of Long Term Evolution (LTE) -Advanced and NGWNs is the use of smaller cells due to the attenuation of radio signals at high frequencies. Unlike $2 \mathrm{G}$ systems where the radius of macro base stations (MBSs) could reach several kilometers, the cell radius of LTE-Advanced and NGWNs such as 5G networks would only be a few hundred meters in order to overcome the radio signal propagation impairments. Small cells are envisioned to increase the path loss due to the shorter range transmission. This concept also brings the higher frequency into the picture. Higher frequencies cannot propagate long distances; therefore, interference to other small cells is likely to be reduced. Therefore, Heterogeneous small cell networks (HetSNets) represent a novel approach for the mobile data offloading problem that helps to optimize the network traffic [3].

HetSNets represent a combination of different size small cells with reduced radius in the range $[10-100] \mathrm{m}$ such as picocells and femtocells with wireless-fidelity (Wi-Fi) access points (APs) and Wi-Fi Direct device-to-device (D2D) enabled links as seen in Fig. 1(a), and are deployed within the existing macrocell. Fig. 1(a) exhibits the provisional picture of the Wi-Fi and D2D supported HetSNets. HetSNets are envisioned to increase the spectral efficiency and enhance the overall network performance by offloading the network traffic from the MBS to small cells [4]. Since small cells operate within a smaller distance compared to MBSs and are attached to the macrocell core network via wireless/wired IP backhaul connections, small cells will provide higher data rates and dedicated capacity to residential areas and hot spots, and reduce the traffic congestion. Small cell base stations (SBSs) have the capability of using the unlicensed RF spectrum where Wi-Fi is currently operating along with the licensed RF spectrum. Such a feature will provide dual mode functionality for SBSs. Therefore, SBSs can provide uninterrupted voice call and text services. At the same time, Wi-Fi satisfies the user's higher demand for multimedia applications such as watching high-definition (HD) videos and online gaming by employing selected IP traffic offloading (SIPTO).

Fig. 1(b) illustrates the anticipated SIPTO traffic offloading (TO) scenarios for the Wi-Fi APs. SIPTO refers to decide whether the mobile user employs SBS or Wi-Fi APs based on the type or content of the traffic-type. Basically, delay tolerant applications will go through Wi-Fi. Wi-Fi Direct protocol on IEEE 802.11 can also be used as a good alternative and will utilize the unlicensed bands on D2D connections where two closely located devices get connected without routing the traffic through the access network [5]. Wi-Fi Direct is the connection protocol which is similar to Bluetooth but it utilizes the Wi-Fi interface. Since some of the users may have the common packets within the vicinity of the same receivers, D2D enabled devices can utilize the shorter radio links from the neighboring devices. This will increase the overall performance of the small cells and reduce the traffic congestion. The recent advances in mmWave communications at $60 \mathrm{GHz}$ spectrum usage for Wi-Fi will bring a new dimension on the coalition between LTE small cells and Wi-Fi APs such as free spectrum usage, less interference, high data rate and reliable low-range transmission [6]. Collaboration between SBSs, Wi-Fi APs/Wireless Gigabit Alliance (WiGig) and D2D enabled devices will significantly reduce the traffic load on the traditional macrocell networks. According to [7], it 
is expected that the total percentage of TO from MBSs to small cells will increase by $60 \%$ by 2020 . Reference [7] describes the TO profile for the countries that already started to deploy small cells. Countries where LTE-advanced and Wi-Fi APs communication technologies have already been implemented present higher TO percentages. Therefore, deployment of Wi-Fi supported small cells are an extremely important task for the network operators.

In this paper, an active user dependent path loss (PL) based TO strategy for HetSNets is proposed and analyzed to overcome the aforementioned problems, where hybrid small cells are distributed homogeneously among the macrocell. The main objective of the PL based strategy is to show the significant difference between signal-to-interference ratio (SIR) based offloading and PL based offloading while considering the AUPF and traffic-type. PL strategy increases the offloading percentage and ensures that more users will transfer to SBS and experience better data throughput and coverage. Further, the offloaded user traffic gets distributed onto SBS, Wi-Fi and D2D enabled links based on the content of the application.

Factors such as population density, access scheme and distribution of small cells across the macrocell area represent challenging issues that can impact the TO directly. In addition to these factors, the daily traffic profile, active user population and traffic-type should also be investigated to optimize the network deployment and QoS. Therefore, the AUPF of mobile users on the traffic offload has also been studied. Due to the aforementioned reasons, answers to the following questions will be addressed herein study:

- What is the impact of hybrid small cell and macrocell radius on the percentage of TO?

- How does the percentage of TO change with respect to the AUPF $(\gamma)$ and the probability of having an open/closed access hybrid small cell?

- How much of the offloaded traffic will go through SBS, Wi-Fi AP and D2D enabled links?

The remainder of this paper is structured as follows. SBS and Wi-Fi APs coalition benefits are discussed in Section 2. A layout of the HetSNets along with the evaluation of the population factor with respect to the number of active small cells and access schemes are depicted in Section 3. PL and SIR TO based strategies are discussed in Section 4, respectively. The TO analysis is presented in Section 5. Section 6 presents economics of TO. Simulation results and discussions are included where required to provide a comparative performance analysis. Finally, the concluding remarks are drawn in Section 7.

1.1 Device-to-Device, Small Cell Base Station and Wi-Fi Based Traffic Offloading

In D2D enabled networks direct transmission through each device is considered. For instance, some devices may utilize the SBSs or Wi-Fi APs for downloading a multimedia file while other close-proximity devices can utilize the shorter direct link to fetch data from its D2D peers like a P2P connection with Wi-Fi Direct or WiGig. This will distribute the traffic load onto D2D enabled devices and reduce the total traffic load. A good example of this situation is that of watching ultra 
HD videos from TV by directly transmitting through a laptop or camera or bluray player. However, D2D enabled devices are battery powered equipment and therefore, the energy consumption will increase and drain the battery of the mobile devices based on the content of the file that is being downloaded or uploaded. This concern can be eliminated by providing higher data rates for the D2D enabled devices. D2D communication does also present reliability concerns due to the rapid $\mathrm{RF}$ environment changes.

Almost $38 \%$ of the total mobile data traffic is offloaded on to Wi-Fi APs and hotspots in US [8] and it is expected to be higher with the implementation of $5 \mathrm{G}$ NGWNs. Since Wi-Fi APs are already deployed at homes, campuses and businesses, it is more convenient for the network operators to utilize the Wi-Fi as the data offloading docking stations. Most of the current cellular phones have the capability of connecting to either Wi-Fi or cellular base stations in order to download multimedia and delay tolerant applications and contents. Mobile devices usually picks the Wi-Fi APs if there is one in the vicinity due to the fact that it will provide higher data rates and reduce the cellular plan cost by utilizing the unlicensed RF spectrum. Especially with the introduction of the WiGig technology which employs the $60 \mathrm{GHz}$ unlicensed and price-free spectrum, it will definitely bring Wi-Fi one step further as the carrier offloading strategy. WiGig operates on a very wide bandwidth which makes the spectrum very attractive for multimedia and delay tolerant applications such as streaming ultra HD from a dvd-player or laptop to a TV set. Especially for backhaul, it creates an opportunity for outdoor point-to-point ( $\mathrm{P} 2 \mathrm{P}$ ) connections to provide Internet access to neighboring buildings to prolong the access of fiber networks. WiGig has several advantages such as (i) intelligent beam control techniques; (ii) energy efficient transmission, i.e., $30 \%$ less energy consumption than LTE small cells; (iii) shorter range and easy interference management; (iv) $7 \mathrm{GHz}$ of free and unlicensed spectrum with Gbps transmission rates; (v) instant wireless synchronization.

Lastly, SBSs are a natural extension of the macrocell network. SBSs provide also a better QoS for voice and delay tolerant applications with no disruption. SBSs utilize the licensed spectrum which enable them to avoid easily unwanted radios and penetrate through buildings. On the other hand, licensed spectrum is limited so $\mathrm{Wi}-\mathrm{Fi}$ will most of the time provide higher data rates. This brings the importance of the multi-homing [9]. Currently, mobile devices are capable of handling higher bandwidth consuming applications along with basic voice and text applications via Wi-Fi and cellular interfaces. Such type of communication presents multi-homing features. Multi-homing associates each radio interface with available overlapped mobile networks which can be cellular base stations or Wi-Fi APs. Therefore, multi-homing has gained significant interest within the past years. Most of the smartphones have the capability of multi-homing which enables each device to connect to any available network as shown in Fig. 1(b). Therefore, after carefully examining these three approaches, one can infer that a combination of these technologies will generate higher data rates, better coverage and less power consumption, a research topic which is currently being investigated in the upcoming Wi-Fi supported 5G NGWNs [10]. Next, the potential benefits of the coalition between SBSs and Wi-Fi will be discussed. 


\section{Small Cell Base Station and Wi-Fi Access Points Coalition Benefits}

The capacity of SBS depends directly on the limited availability of the spectrum which can also be dissipated by the interference from the highly dense SBS deployment. To handle such problems, researchers and network operators started to investigate the usage of Wi-Fi to support the SBSs. Possible integration scenarios can be seen in Fig. 1(a). Integration of these two technologies will bring many benefits:

Radio Frequency Spectrum Utilization: RF spectrum is critical for the wireless communications systems because of its limited availability. Due to the ever increasing demand for it, researchers have sought to find more efficient ways to utilize the RF spectrum. Depending on the design, communication systems use the RF spectrum in different ways. However, one of the most important design features is the fact that wireless systems should optimize the use of resources by employing a set of functionalities. Due to the limited nature of current RF spectrum, the mmWave communication band $60 \mathrm{GHz}$ will bring new degrees of freedoms in terms of TO such as higher data rates with interference immunity, better security and re-use of the frequency bands.

$60 \mathrm{GHz}$ mm Wave Communication: Electromagnetic waves can be absorbed by oxygen molecules while traveling through air. The absorption level is higher at 60 Ghz, therefore, the propagation distance diminishes at such high frequencies. With the great absorption rates in the $60 \mathrm{GHz}$ mmWave communication, e.g., WiGig can be used in shorter range communications to provide very high data rates with no interference to neighboring WiGig devices because of the aforementioned oxygen absorption issue. Moreover, this portion of spectrum is unlicensed so far and will be an attractive option for the operators. However, one interesting point that needs to be addressed is the effect of the rain on the deployment of the outdoor $60 \mathrm{GHz}$ technologies. Rain might dominate the mmWave penetration distance more than the oxygen absorption rate.

Smart Traffic Offloading: The combination of Wi-Fi and SBSs will allow network operators to provide voice and text services through the carrier core network while transferring the Internet traffic through Wi-Fi APs and its backhaul as depicted in Fig. 1(b). This offloading scenario will give the opportunity to use the best of both Wi-Fi and SBSs for the mobile users.

Wi-Fi Backhaul: Small cells typically utilize either the digital subscriber line (DSL), fiber or cable as backhaul connection technology. Small cell owners are not concerned with the type of technology used for backhaul, however, the network operators are concerned by the technology used for backhaul [11]. Stemming from the limited backhaul capabilities, the volume of offloaded traffic from macrocell can be reduced if the small cell backhaul reaches a certain threshold. Therefore, the backhaul constraint can be integrated with the biased user association technique [3]. The biased user association technique refers to the allocation of the mobile user to the available small cells. If the wired backhaul reaches its limits, then Wi-Fi integrated SBSs also enable network operators to use Wi-Fi as a very good candidate for the backhaul transfer. Wi-Fi APs in many SBSs can construct a mesh network for a high quality and dependable wireless backhaul especially with the new WiGig concept which can easily provide fiber optical data transfer rates. Moreover, Wi-Fi can also be used to offload the Machine-type traffic that is envisioned to explode in volume due to the advent of Internet of Things (IoT). 
Cost Efficiency: Network operators have a big craving for successful Wi-Fi and SBS integration in order to eliminate extra operational expenditure (OpEx) and capital expenditures (CapEx) costs for separate installation/planning of the Wi-Fi APs and SBSs. Therefore, dual mode or hybrid small cells which combine the small cell and Wi-Fi on the same hardware will lower the cost of the ownership and increase the usage of shared resources such as backhaul, site rental, spectrum cost and additional equipment. In the earlier years of the small cell deployments, Wi-Fi was considered as a competitor but now it can be easily seen that SBS and Wi-Fi are the two sides of the same coin that can complement each other well. Mutualism of these two technologies in the same box will guarantee the usage of the right technology all the times without contemplation over the radio access technology.

Handover: Seamless handover is a must for the integrated SBS and Wi-Fi technologies due to excessive traffic and interference. It will assure the uninterrupted service quality for the mobile user regardless of the radio interface technology. In these cases, successful offloading which is a part of handover becomes an important issue. One of the easiest ways of incorporating the impact of environment into the handover analysis is to use a simple PL model with different PL exponents corresponding to different environments and cell types.

\section{Heterogeneous Small Cell Network Layout}

In this section, a HetSNets scenario is considered where the small cells are distributed within the macrocell network homogeneously. In terms of the ease of practical implementation, it is assumed that each macrocell is surrounded by six macrocells that generate interference to corresponding mobile users. Moreover, each small cell is encircled by six small cells in order to provide a fair comparison with macrocells.

A circular macrocell of radius $r_{m}[\mathrm{~m}]$ is assigned with a base station (BS), $B_{m}$, which is deployed at the center of macrocell. HetSNets contain $N$ circular small cells of radius $r_{n}[\mathrm{~m}]$ with low power, low cost user deployed BSs, $B_{n}$, which are assumed to be located at the center of each small cell and equipped with a wired/wireless backhaul connection. The number of small cells per macrocell can be expressed as ${ }^{1}[12]$ :

$$
N=\underbrace{\gamma \frac{A_{m}}{A_{n}} \alpha}_{\text {Open Access }}+\underbrace{\gamma \frac{A_{m}}{A_{n}}(1-\alpha)}_{\text {Closed Access }}, 0 \leq \gamma \leq 1,0 \leq \alpha \leq 1,
$$

where $N$ denotes the number of active small cells, and $\gamma$ stands for the AUPF parameter which represents a random variable that controls the small cell population per macrocell and daily traffic profile. The AUPF, $\gamma$, is the ratio of the number of active mobile users, $m_{a}$, and the number of total mobile users, $m_{t}{ }^{2}$. Variable $\alpha$

\footnotetext{
1 The intuition behind this approach is to associate the active user distribution, daily traffic profile and small cell access schemes together.

2 To calculate the AUPF, it is assumed that two types of mobile users exist in the network: (a) active mobile users and (b) inactive mobile users. The number of total mobile users is equal to the total number of active and inactive mobile users, $m_{t}$.
} 
stands for the probability of having an open access small cell. Variables $A_{m}=\pi r_{m}^{2}$ and $A_{n}=\pi r_{n}^{2}$ denote the areas of macrocell and small cell, respectively. Moreover, it is assumed that the small cell is active or inactive based on the probability that a mobile user is active in the vicinity of the small cell. Thereby, the population of the small cells is controlled by the percentile of the active mobile users in a daily traffic profile and access schemes of the small cells.

\subsection{Small Cell Access Schemes}

Based on the privileges and permissions allocated to customers, small cell access schemes can be classified into:

\subsubsection{Closed Access}

Self deployed small cells have a key limitation in a "closed access" scheme since the customers would opt for limiting the access to themselves and their preferred users. As expected, closed access small cells do not enhance the coverage for all customers. Therefore, operators search for better access schemes such as "open access" small cells in order to reduce the OpEx and increase the coverage [13].

\subsubsection{Open Access}

Any random nearby cellular user can utilize the small cells which are in "open access" mode. This will allow the network operator to expand the coverage and network performance but will create additional interference problems. A small cell owner would still favor a closed access scheme in order to employ small cell backhaul and enhance the capacity for particular users [13].

Fig. 2 illustrates ${ }^{3}$ the relationship between $\gamma$ and $\alpha$ with respect to $N$ and $N_{o}$. Variables $N_{o}$ and $N_{c}$ denote the number of active open access small cells and active closed access small cells, respectively. The number of mobile users is assumed to be adaptive in the coverage area. The following parameters are used $r_{m}=400 \mathrm{~m}$ and $r_{n}=20 \mathrm{~m}$ to calculate the total number of small cells per macrocell. Low traffic load presents the probability value $p=0.03$, medium traffic load exhibits $p=0.15$ and high traffic load has $p=0.3$, where $p$ is the probability of having an active user. Fig. 2 demonstrates the impact of variable traffic load profiles on $N$ for different $\alpha$ and $\gamma$. When the traffic load increases, then $N$ takes greater values as expected. For instance, the numerical range of $N$ under low traffic load is the interval $(0,16)$. In case of medium traffic load, the numerical range of $N$ is within the interval $(16,56)$. Also, under high traffic load, $N$ varies in the interval $(56$, 114). As a common observation for Fig. 2, $\alpha$ represents the scaling factor for the daily traffic profile of $N$ and $N_{o}$, where the total number of small cells remains the same while $N_{o}$ takes different values. Another crucial point, a significant role on the number of active small cells is played by AUPF, which is a function of the active mobile user. Therefore, AUPF directly affects $N$ and $N_{o}$ and their values fluctuate with the variable traffic load.

\footnotetext{
3 In Fig. 2 x-axis values are taking decreasing and increasing values in order to show the impact of daily traffic profile. In a 24-hour period, the activity of the mobile users is not monotonically increasing and decreasing.
} 
Deployment of many small size, low cost and low power SBSs and Wi-Fi APs in the existing macrocell network brings into analysis the important function played by TO. When the congested traffic unloads onto the small cells, MBS will experience less congested traffic and perform better. Small cells reduce the transmission distance and increase the data rate and coverage but such a setup will create a disadvantage in terms of SIR for the offloaded users. Users offloaded to smaller cells receive dissipated SIR due to the strongest interference power received. If SIR based offloading takes place, then most of the users will remain in the MBS coverage and receive low data rates and unsatisfactory QoS. Therefore, in this study, the PL based TO is investigated stemming from the fact that the offloaded users onto small cells experience better received power from the closer BSs. This will increase the overall offloading percentage and improve the overall system performance and spectral efficiency. Furthermore, offloaded user traffic is divided between Wi-Fi, D2D and SBSs in order to reduce the traffic congestion of SBSs.

\section{Traffic Offloading Methods: Path loss and Signal-to-Interference Ratio}

\subsection{Path Loss Based Traffic Offloading}

The power of the signal propagating through the environment is considered to carry the distance information between transmitter and receiver. This parameter requires knowledge of several wireless propagation characteristics such as PL and shadowing to obtain an estimate about the distance considered. It is known that wireless propagation in different propagation environments differs from each other. Main reason behind this observation lies in the topographical characteristics of the physical propagation environment. To model the PL in HetSNets where multiple small cells complement the macrocell, several empirical models were proposed in [14, Chapter 12]. However, the measurements indicate that a simple power law PL model cannot be used to fit the measurements with good accuracy especially when the distance between the transmitter and receiver is short $[4,14,15]$. Therefore, this paper considers a two slope (commonly known as dual slope) PL model for HetSNets $[4,14,15]$ : (a) $\mathrm{PL}=K /\left(d^{\zeta_{1}}\right)$ for $d \leq g$, and (b) $\mathrm{PL}=K /\left((d / g)^{\zeta_{2}} g^{\zeta_{1}}\right)$ for $d>g$. Two separate PL exponents, $\zeta_{1}$ and $\zeta_{2}$, which are referred to as basic and additional PL exponents, respectively, are used to characterize two different propagation regions, together with a breakpoint distance $g$ between them where the propagation changes from one regime to the other. The breakpoint of the PL curve is defined as the point after which the strength of the signal attenuates such that the basic PL exponent $\zeta_{1}$ represents the slope before breakpoint and the additional PL exponent $\zeta_{2}$ represents the slope after breakpoint. This can be considered as two regions: for $d \leq g$, i.e., short distances, while for $d>g$, i.e., large distances, where $g=\left(4 h_{r x} h_{t x}\right) /\left(\lambda_{c}\right)[\mathrm{m}]$ is the breakpoint of a PL curve which depends on the MBS and SBS (receiver in uplink) antenna height $h_{r x}[\mathrm{~m}]$, antenna height of the mobile user (transmitter in uplink) $h_{t x}[\mathrm{~m}]$ and wavelength of the carrier frequency $\lambda_{c}$. Parameter $K$ denotes the PL constant.

To avoid the sharp transition between the two regions of a two slope PL model, a generalized propagation model for both macrocell and smallcell networks is considered in $[16,15]$ : 


$$
\mathrm{PL}=\frac{K}{d \zeta_{1}(1+d / g)^{\beta}}, \quad \beta=\zeta_{2}-\zeta_{1}
$$

In order to decide if a user connects to small cell or remains with MBS, the following strategy is assumed. The active mobile user transfers to the small cell if ${ }^{4} \mathrm{PL}_{n}>\mathrm{PL}_{m}$. Otherwise, it remains connected to MBS.

\subsection{Signal-to-Interference Ratio Based Traffic Offloading}

Based on the two slope PL model (2), the received signal power at MBS or SBS from the active mobile user is given by:

$$
P^{r x}=\frac{K}{d \zeta_{1}(1+d / g)^{\beta}} P^{t x} \psi
$$

where

- $P^{r x}[\mathrm{~W}]$ denotes the average received signal power at the reference MBS or SBS from the desired mobile user, located at a distance $d$ from the same reference BS;

- $\psi$ is the composite shadowing and fading component over the link between the mobile user and respective MBS or SBS;

$-P^{t x}[\mathrm{~W}]$ defines the mobile user transmit power for the physical uplink shared channel (PUSCH) such that each mobile user in the macrocell network transmits with the maximum power $P_{\max }$.

SIR is also an important parameter to evaluate the system performance in wireless communications and it is used in many performance metrics such as TO, handover, power control and channel assignment. The general definition of the SIR is the ratio between the average desired received signal power at the reference macrocell or small cell, and the total interference power received from surrounding MBSs or small cells, $P^{r x} / \sum_{i=1}^{6} P_{i}^{r x}$. $P^{r x}[\mathrm{~W}]$ stands for the average desired received signal power at the reference macrocell or smallcell, and $\sum_{i=1}^{6} P_{i}^{r x}[\mathrm{~W}]$ denotes the total interference power received from surrounding MBSs or SBSs. The PL strategy discussed in Section 4.1 can also be applied to SIR based offloading. For instance, if $\operatorname{SIR}_{n}>\mathrm{SIR}_{m}$, then users transfer to SBS. Otherwise, they will continue using MBS.

\section{Traffic Offloading Analysis}

In order to provide more insightful results, the percentage of TO for PL and SIR based strategies is investigated in three different set- $\mathrm{ups}^{5}$ :

\footnotetext{
4 Subscripts $m$ and $n$ are associated with MBS and SBS, respectively.

5 In this study, the simulation results exclusively provide the numbers for MBS to small cell TOs.
} 


\subsection{Cell Size Dependent Traffic Offloading}

In order to point out the impact of cell size on the offloaded traffic, Fig. 3 shows the summary of the radius dependent TO for variable small cell and MBS sizes. It is assumed that $\gamma$ and $\alpha$ are equal to 1 , which means that the macrocell is fully loaded with active mobile users and all the small cells are open access. The active mobile users in the macrocell network receive better SIR than those in the small cell network due to fact that the instantaneous SIR is lower in small cells. On the other hand, since the radius of the small cell is relatively smaller than that of macrocell, it will provide a better PL. Therefore, the significant difference between PL and SIR can be seen in Fig. 3. The impact of TO with respect to the macrocell radius and different values of $r_{n}$ is illustrated in Fig. 3(a). With the increase in $r_{m}$, SIR gets better for the macrocell network due to the reduced interference from the surrounding MBSs. Therefore, the percentage of SIR based offloading decreases in Fig. 3(a). Then again, the PL for the small cell will often be higher and provide at least $78 \%$ TO due to the fact that the radius and the PL exponents are smaller than the values corresponding to the macrocell. For instance, when $r_{m}$ takes values in the interval $(200,1000)$, the percentage of PL based offloading is always greater than $78 \%$ even though $r_{n}$ is increasing from $20 \mathrm{~m}$ to $200 \mathrm{~m}$. This proves that shorter separation leads to a better offloading in terms of PL. As long as there is a drastic ratio between $r_{m}$ and $r_{n}$, PL based offloading will lead to the best performance in terms of TO. Another interesting observation is that $\mathrm{SIR}_{n}$ is getting better when $r_{n}$ takes greater values since the interference from neighboring small cells is decreasing. Fig. 3(b) depicts the PL, SIR and dual mode cognitive based offloading for different $r_{n}$ and $r_{m}$ values. SIR based offloading is increasing as expected due to the fact that $r_{n}$ takes greater values, a factor which reduces the received interference from surrounding small cells. Compared to PL based offloading, the SIR based method provides less TO than the PL based offloading. SIR and PL based offloading strategies take values in these intervals $(24 \%, 57 \%)$ and $(78 \%, 100 \%)$, respectively, indicating that the radii of MBS and small cell are the main factors that affect the TO. For a clearer view of this impact, Table 1 depicts a summary of $N, N_{o}$, TO for low, medium and high traffic loads for different $\alpha$ values, when $r_{n}=20 \mathrm{~m}$ and $r_{m}=400 \mathrm{~m}$.

\subsection{Traffic-Type Dependent Offloading}

Fig. 4 illustrates the percentage of TO onto SBS, Wi-Fi APs or D2D enabled devices. It is assumed that $\gamma$ and $\alpha$ are equal to 1 , which means that the macrocell is fully loaded with active mobile users and all the small cells are open access. It is assumed that TO proportions from the total offloaded small cell traffic to SBSs, Wi-Fi APs and D2D enabled links are given by $54 \%, 38 \%$ and $8 \%$, respectively. For instance, if the total number of traffic offloaded to the small cells is 100 then 54 of them will go through SBS, 38 via Wi-Fi APs and 8 to D2D. In order to provide a better understanding of the TO to SBS, Wi-Fi APs and D2D, Fig. 4 depicts the percentage of the traffic distribution. Since the mobile data traffic is heterogeneous being composed of voice and text transmissions, multimedia applications, P2P connections, social networking applications, etc., it is assumed that voice and text will be associated with SBSs, multimedia and delay tolerant ap- 
plications with Wi-Fi APs and P2P connections with D2D connections [8]. Since voice and text services are of paramount importance for network operators, SBSs will provide uninterrupted QoS. On the other hand, compensation of the higher data requirements will be ensured by Wi-Fi and D2D connections which will also diminish SBS backhaul volume.

\subsection{Daily Traffic Profile and Small Cell Access Scheme Dependent Traffic} Offloading

One of the fundamental reasons for small cell deployment is to reduce the traffic load when the data traffic usage is high and heterogeneous during a 24 hour period. Therefore, Fig. 5 depicts the impact of daily traffic profile and access scheme on TO. Fig. 5 illustrates the percentage of user offloading from MBS to small cell while considering both active and inactive mobile users. Fig. 5(a) demonstrates the effects of $\alpha$ for different $\gamma$ values. As expected, the slope of the curve almost stays constant, which illustrates that $\gamma$ is the main factor that alters the percentage of TO. For low traffic values, the percentage of TOs takes values in the interval $(0 \%$, $3 \%$ ) when $\alpha$ is increasing from 0 to 1 . In case of high traffic load, the percentage of offloadings ranges in the interval $(0 \%, 30 \%)$. Fig. 5(b) depicts the impact of $\gamma$ on the percentage for different $\alpha$ values. The curve of the offloading percentage changes drastically for different $\gamma$ values. It is evident that the traffic load decreases when the percentage of the offloaded traffic reduces due to the diminishing number of active mobile users and small cells. As an example, low, medium and high traffic loads are chosen. The percentage of offloading takes values in the intervals $(0 \%$, $3 \%),(3 \%, 12 \%)$, and $(12 \%, 21 \%)$, respectively.

\section{Economics of Traffic Offloading}

Network operators' exhibit special interest into TOs through proper placement of small cells since it is a necessity to reduce the total cost of ownership (TCO), CapEx which refers to the equipment and installation cost and OpEx which refers to the cost to keep the system running. Site lease and transportation generates the biggest chunk of the total cost in the network planning. In traditional macrocell network, placement of a new cellular tower in a highly dense location i.e., downtown, can be impossible. Therefore, utilizing the already existing infrastructure and third party solutions such as Wi-Fi, femtocells and D2D communications, are anticipated to reduce the total cost. Data offloading through Wi-Fi and femtocells has a great value in different dimensions of a business such as hotspot operators, manufacturing devices and service providers. The greatest benefit in terms of cost savings is reducing the OpEx. First, operators only need to add little or no upgrades for the backhaul traffic due to the already existing backhaul infrastructures such as DSL lines, fiber, etc., for the TO which will relieve the congested traffic. Second, there is no need for drastic changes in the hardware and software for the radio access network. In a macrocell network, the cost per GB-RAN equals $\$ 21.80$, while it is only $\$ 8.19$ for the Wi-Fi supported SBSs [17]. Therefore, both LTE SBSs and Wi-Fi APs have shown significant CapEx and OpEx reductions opposed to 
LTE macrocells such as $85 \%$ decrease taking into account both CapEx and OpEx $[18]$.

Reference [18] showed TCOs for scenarios where SBSs are cheaper for low density buildings. On the other hand, Wi-Fi APs dominate the TCO with an increase in the density. SBSs are almost $31 \%$ cheaper for low density buildings while Wi-Fi APs are cheaper $60 \%$ for higher density buildings. One can interpret Fig. 1(b) for the smart TO. If hybrid offloading takes place, it might further reduce TCO. Despite the fact that $60 \mathrm{GHz}$ mmWave communications are still under maturation stage, they will bring a huge offloading relief for the indoor communications and backhaul traffic. Operators can easily build a mesh network with $60 \mathrm{GHz}$ devices to eliminate the crowded usage of the wired backhaul connections. Another important aspect of the cost reduction is for the mobile subscribers. Since the current data plans for cellular 3G/4G networks are considerably high and limited per month, Wi-Fi offloading in the residential areas diminishes the data usage and increase savings for each bill cycle. The mobile operator's business is to provide data services and they are supposed to generate revenues by offering such a service. One may wonder about the motivation of reducing the data load on a mobile operator's data network by offloading it to Wi-Fi and small cells technologies which is equivalent to a scenario where a vendor willingly reduces the volume of the sale in a business. The reason for this motivation of an operator is evident when the current revenue models are considered. Users are keen to get data services and faster data rates but the cost that they are willing to pay is limited. This has resulted in several flat-charge models for the data services in mobile service contracts in Europe, America and many Asian countries. In this flat-charge model, the mobile operator does not charge the customer for the "amount of the data", rather it charges the customers a fixed monthly rate for the "offering of the service", when and where needed. Hence, if these operators have a choice to seamlessly offload some of the users to other offloading technologies that are not managed by the operator, this will free up some user and data capacity in operator's system and will allow the operator to enroll more customers with a contract of "offering the service". These flat-charge models are one of the reasons for saturating average revenue per user (ARPU) for the operators. In short, in the presence of a flatcharge billing model the operator is not interested in serving a larger volume of data to each customer; rather the focus is on enrolling as many users as possible while ensuring that they have an experience of good service offering when they need the service in absence of any other alternative source.

Based on this information, the aforementioned strategies in this study can shrink the total costs. Therefore, adding low cost Wi-Fi supported small cells will decrease the marginal cost for network operators and mobile subscribers, reduce the number of sites per network and expand the network coverage. Lastly, the reduction of the number of active cells per network will yield huge energy savings due to the fact that small cells are using very low power compared to the traditional macrocell. Even though, small cells are low cost devices but a huge number of installed small cells can significantly increase the total cost, therefore, the number of small cells is an issue that still needs to be carefully planned. 


\section{Concluding Remarks}

HetSNets have gained significant attention due to their unique characteristics. Many studies are currently conducted to utilize this new concept more efficiently in $4 \mathrm{G}$ and $5 \mathrm{G}$ NGWNs. Due to the size and cost constraints on placing a new MBS in the existing cellular networks, it is wise to use low cost and highly spectrally efficient Wi-Fi and D2D enabled links supported small cells. One of the main objectives of HetSNets is to help reduce the traffic load in the existing MBSs. Therefore, this study investigated the effect of daily heterogeneous traffic profile and exploited it in the PL based TO strategy where variously sized small cells are spread over the macrocell homogeneously. It is shown that changes in traffic load profiles and probability of having an active mobile user lead to changes on the number of active smallcells, as expected. It is also demonstrated that the heterogeneous nature of the traffic-type is also an important and useful parameter to spread the congested traffic over the various types of technologies such as SBSs, Wi-Fi APs and D2D enabled links. Delay tolerant applications can use Wi-Fi APs and D2D enabled links where voice/text service can go through SBSs. This will not only reduce the over-the-air traffic but also truncate the wired backhaul bottleneck problem with the integration of Wi-Fi and D2D communication technologies. In order to illustrate the improvements, the SIR based offloading is compared with the PL based method. It is shown that substantial offloading improvements can be achieved with the hybrid TO strategy, which allows for a reduction in the congested macrocell traffic. If the radius of the small cell is shorter than that of macrocell, then PL based offloading will most likely provide higher offloading percentages than SIR based offloading in terms of transferring congested traffic. This reduces the over-the-air signaling, operational and capital expenditures, and will increase the spectral efficiency, data rates and coverage.

\section{References}

1. G. Demestichas, A. Georgakopoulos, D. Karvounas, K. Tsagkaris, V. Stavroulaki, J. Lu, C. Xiong, and J. Yao, " $5 \mathrm{G}$ on the Horizon: Key Challenges for the Radio-Access Network," IEEE Veh. Technol. Mag., vol. 8, no. 3, pp. 47-53, 2013.

2. M. Z. Shakir, K. A. Qaraqe, H. Tabassum, M. S. Alouini, E. Serpedin, and M. A. Imran, "Green heterogeneous small-cell networks: toward reducing the $\mathrm{CO} 2$ emissions of mobile communications industry using uplink power adaptation," IEEE Commun. Mag., vol. 51, no. 6 , pp. 52-61, 2013.

3. A. Ghosh, N. Mangalvedhe, R. Ratasuk, B. Mondal, M. Cudak, E. Visotsky, T. A. Thomas, J. G. Andrews, P. Xia, H. S. Jo, H. S. Dhillon, and T. D. Novlan, "Heterogeneous Cellular Networks: From Theory to Practice," IEEE Commun. Mag., vol. 50, no. 6, pp. 54-64, 2012.

4. M. Z. Shakir and M.-S. Alouini, "On the area spectral efficiency improvement of heterogeneous network by exploiting the integration of macro-femto cellular networks," in Proc. IEEE Intl. Conf. on Commun. Workshops (ICC'12), Ottawa, Canada, Jun. 2012, pp. 5695-5700.

5. "IEEE, Std 802.11-2012: Wireless LAN Medium Access Control (MAC) and Physical Layer (PHY) Specifications," 2012.

6. S. Rangan, T. S. Rappaport and E. Erkip "Millimeter-Wave Cellular Wireless Networks: Potentials and Challenges," Proceedings of the IEEE, vol. 102, no. 3, pp. 366-385, 2014.

7. CISCO, "Cisco Visual Networking Index: Global Mobile Data Traffic Forecast Update, 2013-2018," http://www.cisco.com/en/US/solutions/collateral/ns341/ns525/ ns537/ns705/ns827/white_paper_c11-520862.pdf, 2014

8. COMSCORE, "How Tablets, Smartphones and Connected Devices are Changing U.S. Digital Media Consumption Habits," http://www.ipmark.com/pdf/Omnivoros.pdf, 2011. 
9. M. Ismail and W. Zhuang, "A distributed multi-service resource allocation algorithm in heterogeneous wireless access medium," IEEE J. Sel. Areas Commun., vol. 30, no. 2, pp. 425-432, 2012

10. M. Bennis, M. Simsek, A. Czylwik, W. Saad, S. Valentin, and M. Debbah, "When Cellular Meets WiFi in Wireless Small Cell Networks," IEEE Commun. Mag., vol. 51, no. 6, pp. 44$50,2013$.

11. O. Tipmongkolsilp, S. Zaghloul, and A. Jukan, "The Evolution of Cellular Backhaul Technologies: Current Issues and Future Trends," IEEE Commun. Surveys \& Tuts., vol. 13, no. 1, pp. $97-113,2011$.

12. A.R. Ekti, M. Z. Shakir, E. Serpedin and K.A. Qaraqe, "Downlink power consumption of HetNets based on the probabilistic traffic model of mobile users," in Proc. IEEE 24th Intl. Symp. on Personal Indoor and Mobile Radio Commun. (PIMRC'13), London, United Kingdom, Sep. 2013, pp. 2797-2802.

13. G. de la Roche, A. Valcarce, D. Lopez-Perez, and J. Zhang, "Access control mechanisms for femtocells," IEEE Commun. Mag., vol. 48, no. 1, pp. 33-39, January 2010.

14. S. R. Saunders, and A. A. Zavala, Antenna and Propagation for Wireless Communication Systems, 2nd ed. Chicester, UK: John Wiley \& Sons Ltd., Mar. 2007.

15. I. Rodriguez, H. C. Nguyen, N. Jorgensen, T. B. Sorensen, J. Elling, M. B. Gentsch, and P. Mogensen, "Path Loss Validation for Urban Micro Cell Scenarios at $3.5 \mathrm{GHz}$ Compared to $1.9 \mathrm{GHz}$," in Proc. of the IEEE Global Commun. Conf. (GLOBECOM'13), Atlanta, GA, Dec. 2013

16. P. Harley, "Short distance attenuation measurements at $900 \mathrm{MHz}$ and $1.8 \mathrm{GHz}$ using low antenna heights for microcells," in IEEE J. Sel. Areas Commun., vol. 7, no. 1, pp. 5-11, Jan. 1989.

17. M. Paolini, "Cost savings and revenue benefits from Next Generation Hotspot (NGH) Wi-Fi," http://www.wballiance.com/wba/wp-content/uploads/downloads/2013/ 09/Cost-savings-and-revenue-benefits-from-NGH.pdf, 2013.

18. J. Madden, "Economic case studies of small cells
and madden-economic-case-studies-small-cells-and-wi-fi/2013-06-28, 2013. 


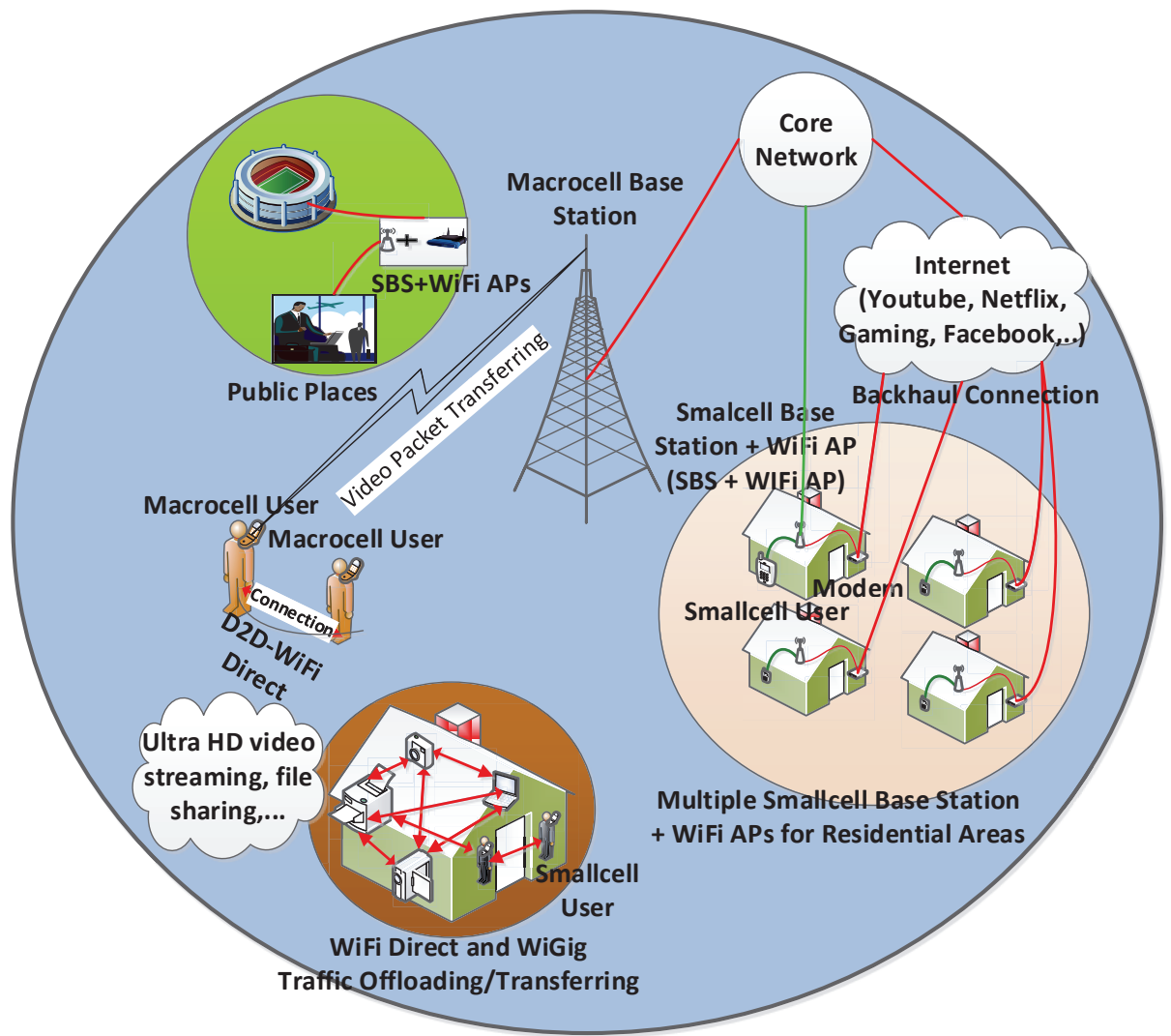

(a) Small cell and Wi-Fi possible connection types

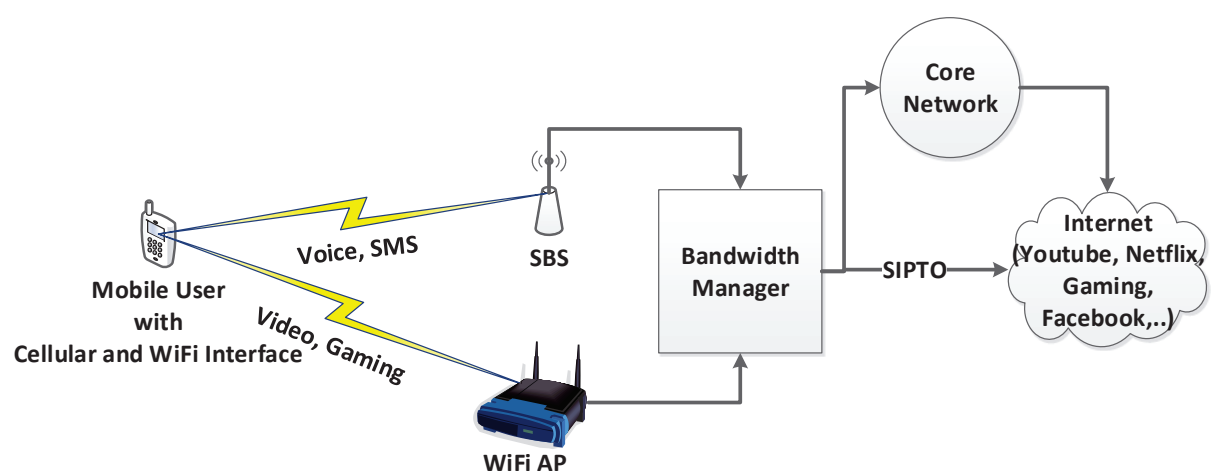

(b) Selective offloading

Fig. 1: Graphical illustration of Wi-Fi and Small cell coalition and selective offloading. 


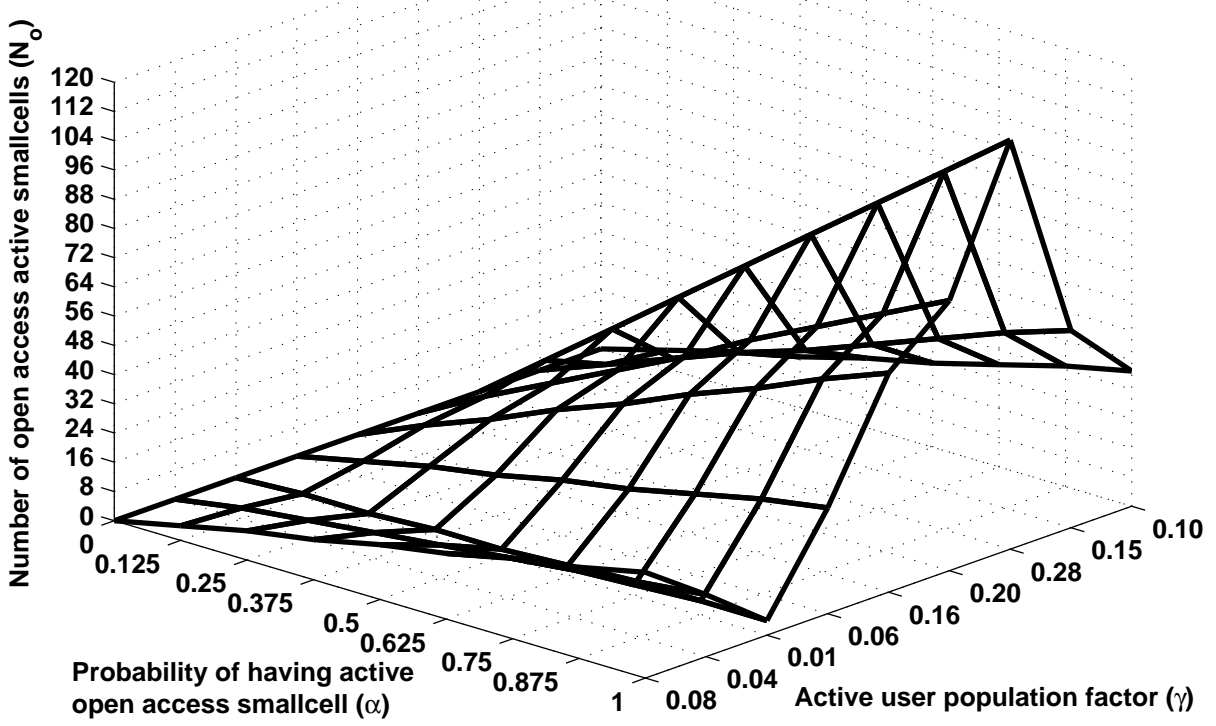

Fig. 2: Number of small cells per macrocell as a function of active user population factor and probability of having open access small cell for daily traffic profile. 


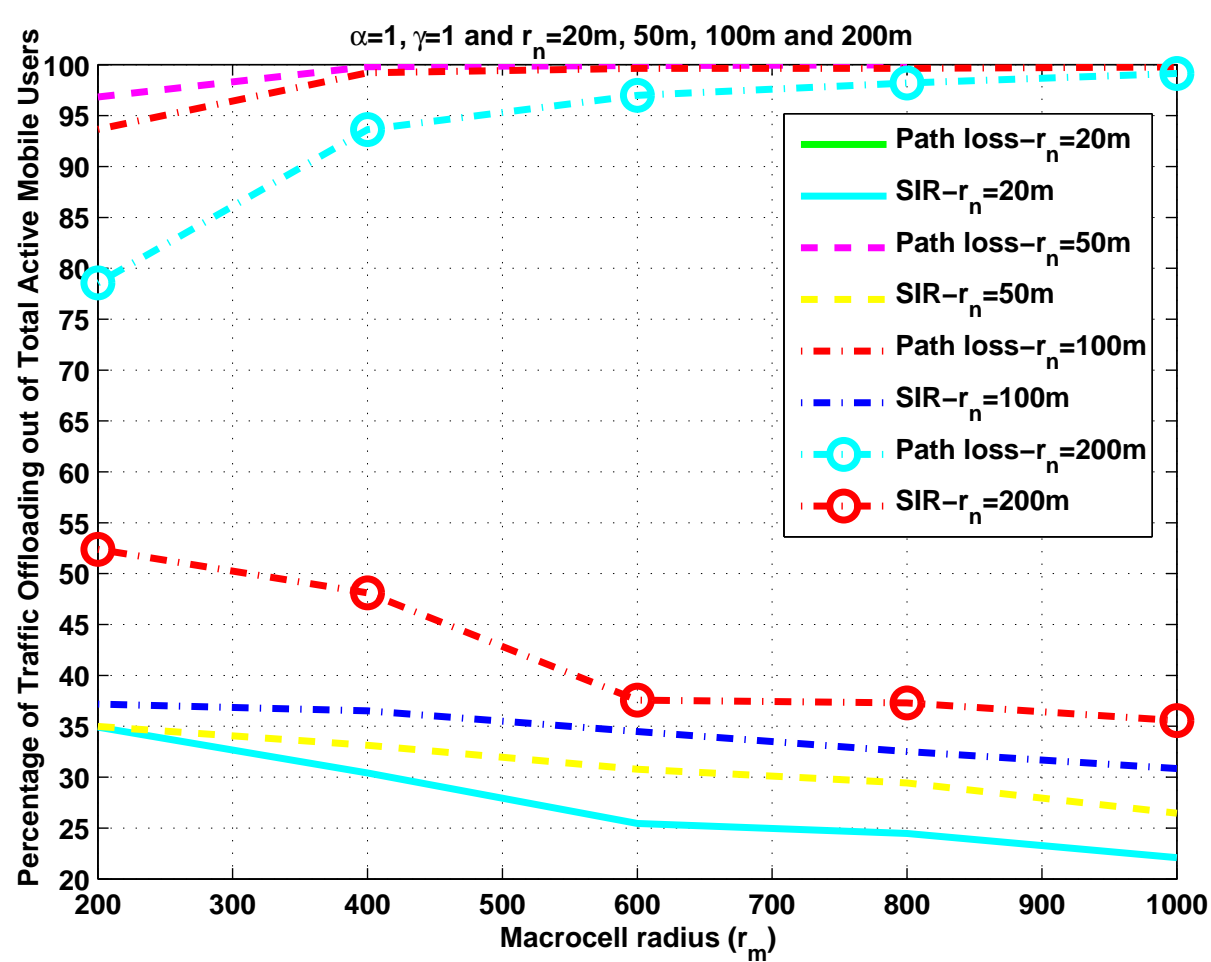

(a)

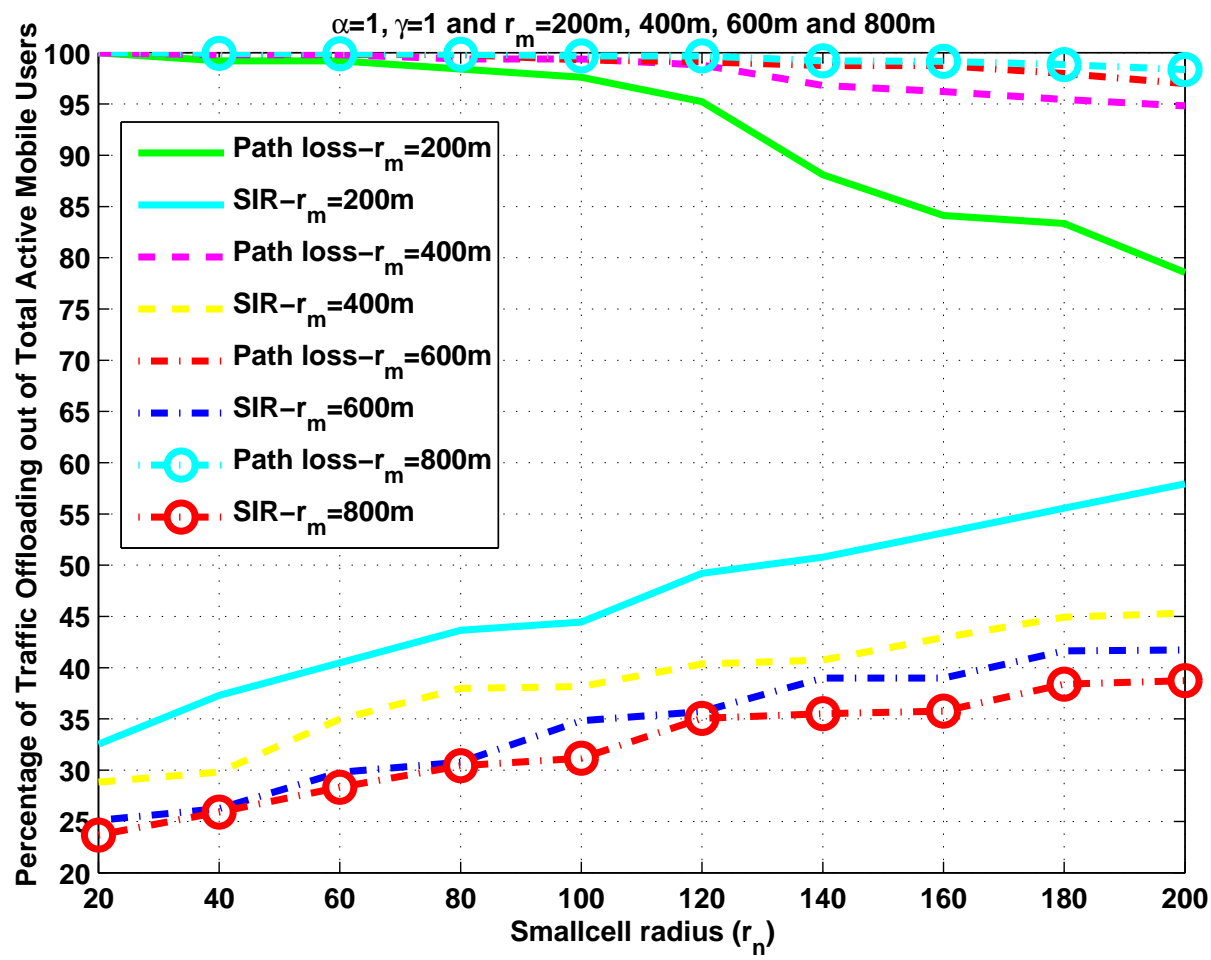

(b)

Fig. 3: Percentage of an active mobile user only traffic offloadings (TOs) per macrocell as a function of active user density per $\mathrm{m}^{2}$, radius of macrocell and radius of small cell. 


\begin{tabular}{|c|c|c|c|c|c|c|c|}
\hline \multirow{8}{*}{ 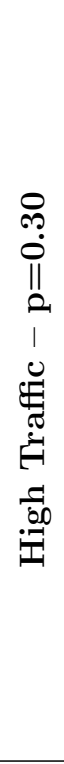 } & \multirow{3}{*}{$\vec{a}$} & $\begin{array}{l}\mathscr{n} \\
\tilde{O}\end{array}$ & 0 & คิ & 악 & 10 & $\stackrel{10}{1}$ \\
\hline & & $\begin{array}{l}\overrightarrow{1} \\
\overrightarrow{1} \\
\overrightarrow{3}\end{array}$ & 0 & $\stackrel{10}{-1}$ & $\stackrel{\infty}{N}$ & 129 & $\overline{0}$ \\
\hline & & Aิ & 0 & $\infty$ & $\infty$ & $\stackrel{\mathcal{I}}{\sim}$ & $\stackrel{10}{-1}$ \\
\hline & \multirow{3}{*}{$\stackrel{n}{n}$} & $\stackrel{\mathscr{n}}{\boldsymbol{n}}$ & 0 & 0 & $\stackrel{\mathcal{N}}{-}$ & คิ & $\stackrel{\sim}{N}$ \\
\hline & & $\frac{\vec{x}}{1}$ & 0 & 10 & 0 & $\stackrel{12}{-1}$ & $\stackrel{0}{-1}$ \\
\hline & & $\begin{array}{l}\text { Aิ } \\
\text { คे }\end{array}$ & 0 & $N$ & $\infty$ & 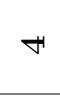 & 10 \\
\hline & \multicolumn{2}{|c|}{$z^{u}$} & $\underset{\mathcal{I}}{\mathcal{I}}$ & $\sigma$ & 6 & $\bar{n}$ & 0 \\
\hline & \multicolumn{2}{|c|}{$z^{0}$} & 0 & ले & 6 & $\sigma$ & $\underset{\mathcal{N}}{\mathcal{N}}$ \\
\hline \multirow{8}{*}{ 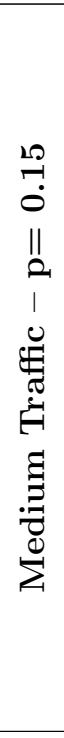 } & \multirow{3}{*}{$\vec{a}$} & 只 & 0 & $\stackrel{-}{\circ}$ & คิ & $\stackrel{\infty}{N}$ & $\hat{\infty}$ \\
\hline & & $\stackrel{\vec{I}}{\vec{I}}$ & 0 & N & $\stackrel{12}{\rightarrow}$ & คึ & คి \\
\hline & & $\begin{array}{l}\text { Aิ } \\
\stackrel{\hat{\theta}}{ }\end{array}$ & 0 & $N$ & $\infty$ & 0 & $\infty$ \\
\hline & \multirow{3}{*}{$\stackrel{\mathscr{n}}{\boldsymbol{\sigma}}$} & $\begin{array}{l}\tilde{N} \\
\boldsymbol{m}\end{array}$ & 0 & $\nabla$ & 0 & $=$ & $\stackrel{\infty}{\sim}$ \\
\hline & & 仝 & 0 & $N$ & $\infty$ & $\infty$ & $\stackrel{\circ}{-1}$ \\
\hline & & คิ & 0 & -1 & -1 & $\boldsymbol{N}$ & N \\
\hline & \multicolumn{2}{|c|}{$z^{u}$} & 8 & $\stackrel{12}{f}$ & मे & $\stackrel{10}{-1}$ & 0 \\
\hline & \multicolumn{2}{|c|}{$z^{\circ}$} & 0 & $\stackrel{10}{-1}$ & के & $\stackrel{10}{7}$ & $\stackrel{8}{0}$ \\
\hline \multirow{8}{*}{ 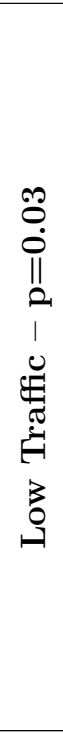 } & \multirow{3}{*}{$\vec{a}$} & $\begin{array}{l}\tilde{m} \\
\tilde{m}\end{array}$ & 0 & $N$ & $\nabla$ & 0 & $\wedge$ \\
\hline & & $\frac{\dot{I}}{i}$ & 0 & -1 & $\infty$ & 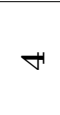 & 0 \\
\hline & & คิ & 0 & -1 & -1 & $\boldsymbol{N}$ & N \\
\hline & \multirow{3}{*}{$\underset{\sim}{\mathscr{E}}$} & $\begin{array}{l}\boldsymbol{N} \\
\boldsymbol{n}\end{array}$ & 0 & -1 & $\boldsymbol{N}$ & $N$ & $\boldsymbol{N}$ \\
\hline & & $\vec{I}$ & 0 & 0 & -1 & -1 & N \\
\hline & & คิ & 0 & 0 & 0 & -1 & -1 \\
\hline & \multicolumn{2}{|c|}{$z^{u}$} & $\stackrel{\mathcal{F}}{-}$ & 0 & 0 & $\infty$ & 0 \\
\hline & \multicolumn{2}{|c|}{$z^{\circ}$} & 0 & $\infty$ & 0 & 0 & $\stackrel{\mathcal{N}}{\sim}$ \\
\hline & \multicolumn{2}{|l|}{$\gamma$} & 0 & 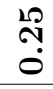 & $\begin{array}{l}0 \\
0 \\
0\end{array}$ & $\stackrel{10}{0}$ & -1 \\
\hline
\end{tabular}

Table 1: Numerical Values of the Number of Traffic Offloading (TO), Number of Open-Access small cells and Number of Closed-Access small cells. 


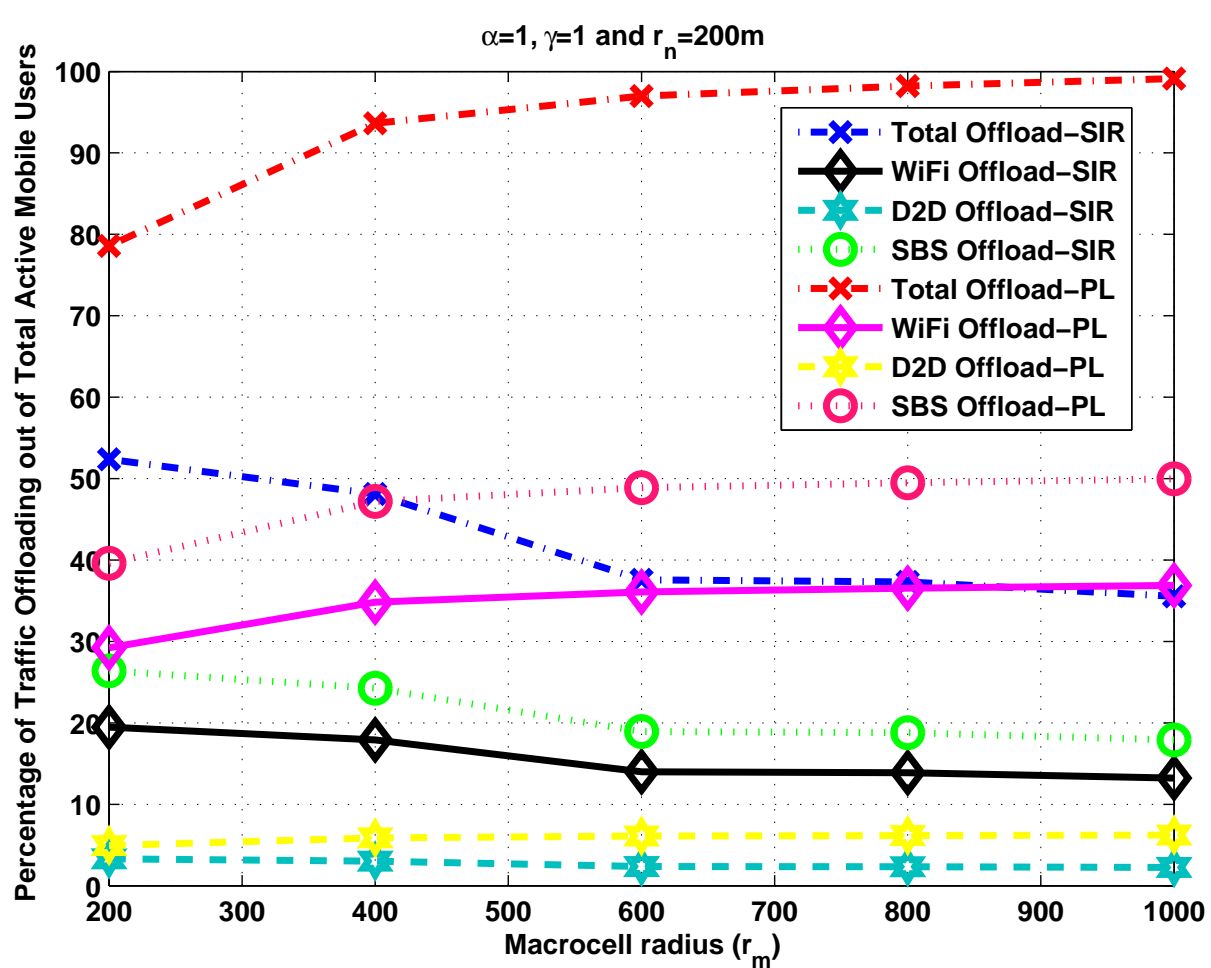

(a)

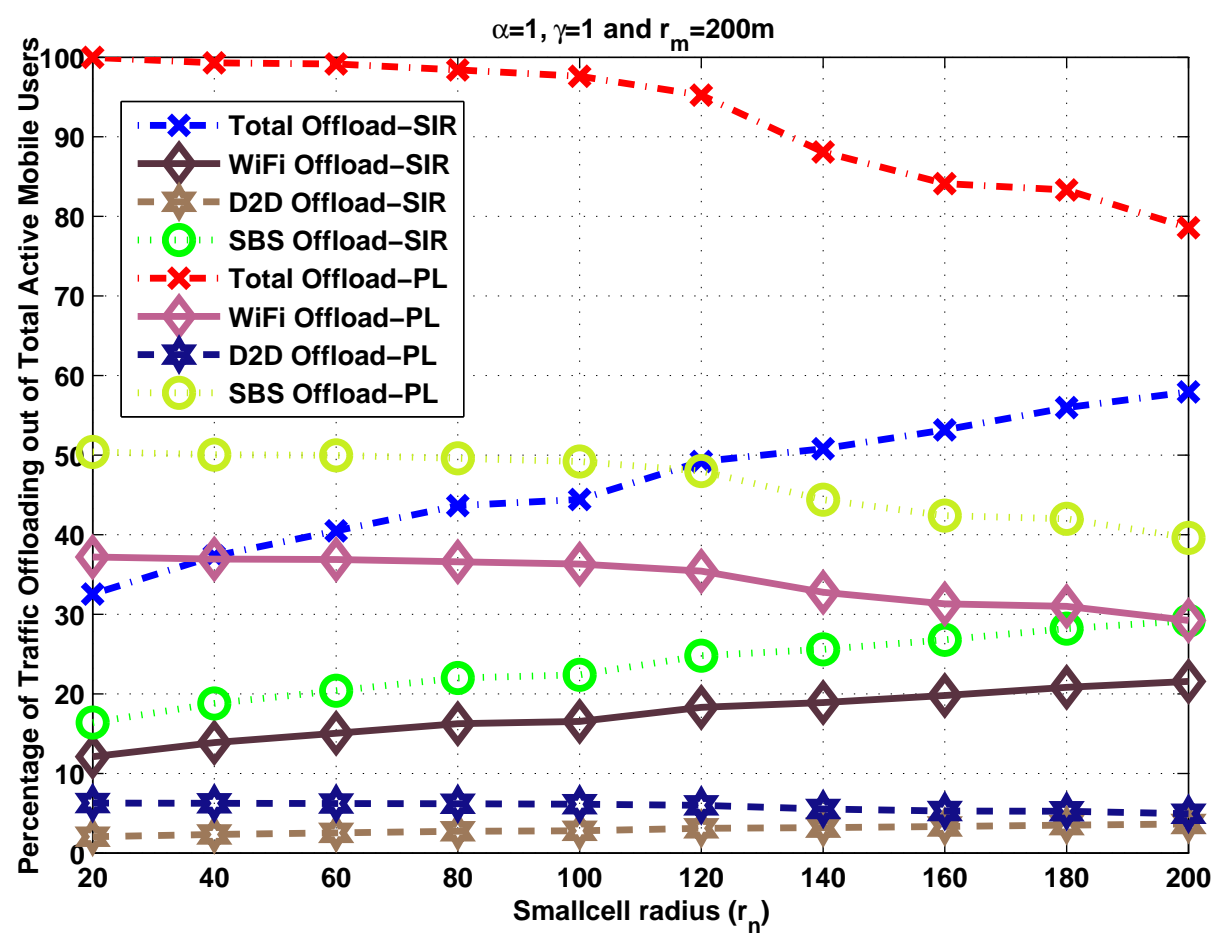

(b)

Fig. 4: Percentage of an active mobile user only traffic offloadings (TOs) to small cells per macrocell as a function of traffic-type. 


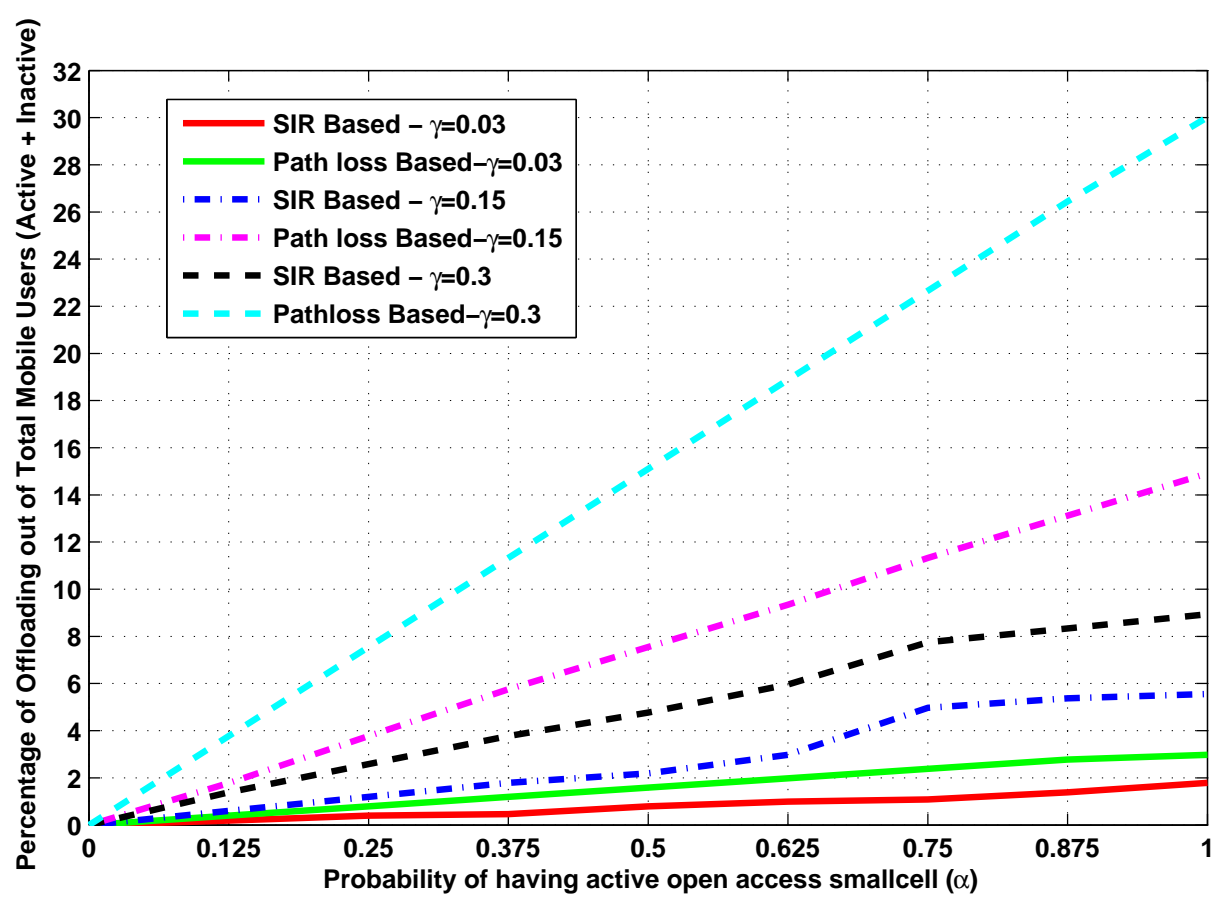

(a)

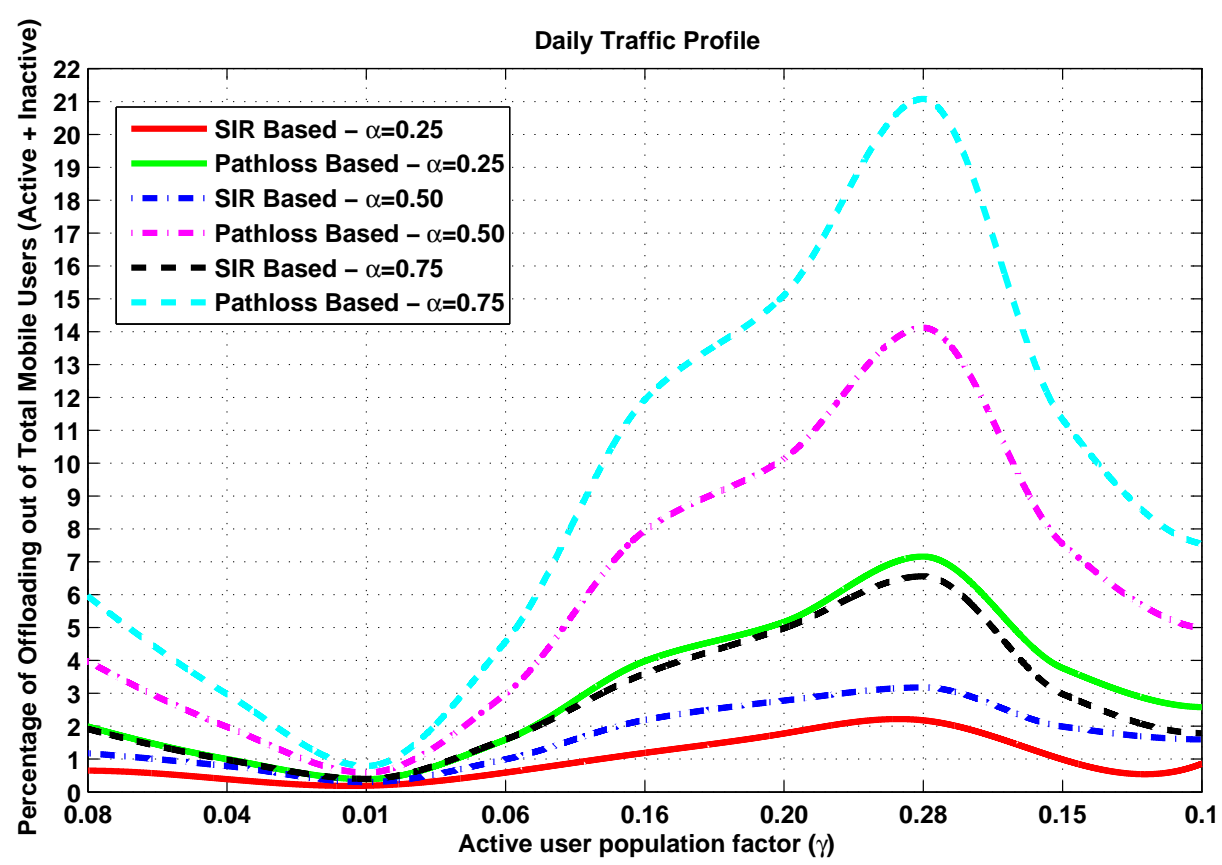

(b)

Fig. 5: Percentage of total traffic offloading (TO) per macrocell as a function of active user population factor and the probability of having an open access small cell. 